\title{
Seroprevalence of SARS CoV-2 infection in Low and Middle-Income Countries and risk classification in the context of rolling out vaccine candidate across these countries : Protocol for a systematic review and meta-analysis
}

Martin Ackah

Korle Bu Teaching Hospital

Louise Ameyaw

Achimota Government Hospital

Kwadwo Owusu Akuffo

Kwame Nkrumah University of Science and Technology College of Science

Cynthia Osei Yeboah

Korle Bu Teaching Hospital

Nana Esi Wood

Nsawam Government Hospital

Rachidatou Compaore

Institute of Health Sciences Research

Denny John

Amrita Institute of Medical Sciences and Research Centre: Amrita Institute of Medical Sciences

Paolo Eusebi

Regional Health Authority of Umbria

Narcisse Tounaikok

Emi Koussi University

Mohammed Gazali Salifu

Ghana Ministry of Health

Anthony Danso-Appiah ( $\nabla$ tdappiah01@gmail.com)

University of Ghana School of Public Health https://orcid.org/0000-0003-1747-0060

\section{Research Article}

Keywords: Seroprevalence, LMICs, SARS-COV-2, Antibodies, Low and middle-income countries, systematic review, meta-analysis 
Posted Date: June 1st, 2021

DOl: https://doi.org/10.21203/rs.3.rs-578481/v1

License: (c) (i) This work is licensed under a Creative Commons Attribution 4.0 International License. Read Full License 


\section{Abstract}

Background Seroprevalence of SARS Cov-2 provides a good indication of the extent of exposure and spread in the population, as well as those likely to benefit from a vaccine candidate. To date, there is no published or ongoing systematic review on the seroprevalence of COVID-19 in Low- and Middle-Income Countries (LMICs). This systematic review and meta-analysis will estimate SARS Cov-2 seroprevalence and the risk factors for SARS Cov-2 infection in LMICs.

Methods We will search PubMed, EMBASE, WHO COVID-19 Global research database, Google Scholar, the African Journals Online, LILAC, HINARI, medRxiv, bioRxiv and Cochrane Library for potentially useful studies on seroprevalence of COVID-19 in LMICs from December 2019 to December 2020 without language restriction. Two authors will independently screen all the articles, select studies based on prespecified eligibility criteria and extract data using a pre-tested data extraction form. Any disagreements will be resolved through discussion between the authors. The pooled seroprevalence of SARS CoV-2 for people from LMICs will be calculated. Random effects model will be used in case of substantial heterogeneity in the included studies, otherwise fixed-effect model will be used. A planned subgroup, sensitivity and meta-regression analyses will be performed. For comparative studies, the analyses will be performed using Review Manager v 5.4; otherwise, STATA 16 will be used. All effect estimates will be presented with their confidence intervals.

Discussion The study will explore and systematically review empirical evidence on SARS Cov-2 seroprevalence in LMICs, and to assess the risk factors for SARS Cov-2 infection in Low Middle Income Countries in the context of rolling out vaccines in these countries. Finally, explore risk classifications to help with the rolling out of vaccines in LMICs.

Systematic review registration: The protocol for this review has been registered in PROSPERO (CRD422020221548).

\section{Background}

In 2020 and potentially beyond, the burden of the novel Severe Acute Respiratory Syndrome Coronavirus 2 (SARS-CoV-2) has been a serious public health and security threat [1]. "Coronaviruses are large, enveloped, positive-strand RNA viruses that can be categorized into genera; alpha, beta, delta and gamma, of which alpha and beta are known to infect humans" [2, 3]. Four Human Corona Viruses (HCoVs) i.e. HCoV 229E, NL63, OC43 and HKU1 are endemic globally and account for $10 \%-30 \%$ of upper respiratory tract infections in adults humans $[2,4]$. The COVID-19 is by far the largest outbreak of atypical pneumonia since the severe acute respiratory syndrome (SARS) outbreak in 2003 in Asia [5]. Generally, about $10-15 \%$ of patients infected with SARS-Cov-2 need hospitalization and $20-30 \%$ of hospitalized patients suffer substantial or life-threatening complications of COVID-19 [6, 7].

It is estimated that nearly $40-45 \%$ of the SARS CoV- 2 infection is symptomless [8]. Thus, monitoring the pandemic escalation by $\lg \mathrm{G}$ [9] and IgM is essential. IgM and IgG can provide a reliable indicator of 
SARS-CoV-2 exposure and immune status of the population $[1,10,11]$ to inform on effective clinical intervention strategies. Undoubtedly, tracking immunity and exposure level patterns in the population is of utmost importance both in the economically advantaged and disadvantaged nations [12]. However, there is paucity of data and thus little understanding of the dynamics and existence of immune response to SARS Cov-2 infection especially in Low- and Middle-Income Countries (LMICs), and scientific basis for lasting immunity on which public health and clinical strategies can be based, is not well described [13].

A recent worldwide seroprevalence study estimated that COVID-19 seroprevalence ranged from $0.4-22 \%$ (translating to 31 million to 1.7 billion people who have been exposed to the infection so far), with a pooled prevalence of $3 \%$ (translating to 234 million people who have been exposed to the infection so far in the general population [1]. This suggest that still over $90 \%$ of the World's population have not got the infection and are susceptible to acquiring the infection. The small number of COVID-19 reinfection to date and history of natural infection with other viral diseases indicate that protective immunity to SARS CoV-2 is a prerequisite for a successful vaccine [14]. In LMICs and the imminent launch of SARS CoV-2 vaccine, it is essential to estimate exposure and degree of spread of the COVID-19 in these countries to at least know the numbers of people likely to benefit from the vaccine (15).

Due to limited socio-economic population status and resources that exist in LMICs [16], essential public health intervention (i.e., lockdown, social distancing etc.) were difficult to put in place or sustain. These, coupled with a limited testing capacity for COVID-19, hinders effective and efficient "public health response planning" [16] This highlights the fact that LMICs are likely to underestimate the true prevalence and incidence of SARS Cov-2 infection. To date, there are no published or ongoing systematic review on seroprevalence of COVID-19 in LMICs.

The objective of this study is to systematically synthesize empirical evidence on COVID-19 seroprevalence in LMICs and risk classifications to help inform sound decision-making about control options, and most importantly, rolling out of vaccine in these economically disadvantaged countries

\section{Methods/design}

\section{Protocol Registration and best practices}

This protocol has been registered with International Prospective Register of Systematic Reviews (PROSPERO) database with registration number CRD422020221548 and reported in compliance with Preferred Reporting Items for Systematic review and Meta-analyses Protocol (PRISMA-P) checklist [17] [see checklist in supplementary file 1] and Meta-analysis of Observational studies in Epidemiology (MOOSE) [18]. [see checklist in supplementary file 2].

\section{Review questions}

1. What is the estimated seroprevalence of SARS CoV-2 infection in LMICs? 
2. What are the risk factors for SARS CoV-2 infection in LMICs in the context of rolling out vaccines in these countries?

3. How can risk classifications help with the rolling out of vaccines in LMICs?

\section{Criteria for considering studies in the review}

\section{Types of studies}

Observational studies including cross-sectional, case-control and cohort studies reporting seroprevalence of SARS Cov-2 infection in any LMIC as defined by World Bank Group will be eligible for inclusion. Animal studies, reviews, commentaries and letter to the editor as well as seroprevalence studies reporting SARS Cov-2 infection from high-income countries will be excluded.

\section{Participants}

Seroprevalence studies involving population from LMICs whose previous COVID-19 infection status is unknown. The review will consider all age groups (children, adolescents and adults). SARS Cov-2 Seroprevalence will be determined by the presence of IgM and/ or IgG antibodies as measured by the serological methods. Suspected SARS CoV-2 participants will be excluded.

\section{Outcomes}

\section{Primary outcome}

The primary outcome in this systematic review and meta-analysis is the seroprevalence of SARS Cov-2 infection in LMICs. Where necessary, this will be sub-grouped by age, sex, socio-economic status or comorbidity.

\section{Secondary outcome}

To assess the risk factors for SARS Cov-2 infection in LMICs in the context of rolling out vaccines in these countries.

\section{Data source and Search strategies}

In collaboration with an experienced medical librarian, we will search electronic databases including PubMed, EMBASE, WHO COVID-19 Global research database, Google Scholar, African Journals Online, LILAC, HINARI, medRxiv, bioRxiv and Cochrane Library for relevant studies on seroprevalence of COVID-19 in LMICs from January to December 2020, without language restriction. The search terms will include; SARS Cov-2 seroprevalence, LMICs, ELISA and LMICs (see Table 1 for details). References of retrieved studies will be hand searched to ensure relevant papers are not missed. In addition, grey literature such as dissertation databases will also be accessed through online university repositories, conference proceedings will be searched, and experts will be contacted to ensure that potentially relevant studies that will be missed by the search of electronic databases are captured. 
Table 1

Search strings for electronic database

\section{Search Searches \\ \#}

1. Seroprevalence OR antibody OR ELISA

2. "COVID-19"[Mesh] OR "2019-nCoV"[Mesh] OR "severe acute respiratory syndrome coronavirus 2"[Mesh] OR "SARS-CoV-2"[Mesh] OR "Wuhan coronavirus" OR 2019 novel coronavirus* OR "coronavirus disease 2019 virus"* OR "COVID19 virus"* OR "2019-nCoV infection" OR "novel coronavirus pneumonia"*

3. Asia OR Africa OR Caribbean OR "Central America" OR "South America"

4. low- and middle-income countr* OR low and middle income countr* OR low- and middleincome nation*OR low and middle income nation* OR low- and middle-income world OR low and middle income world OR low- and middle-income econom* OR low and middle income econom* OR low income countr* OR middle income countr* OR low-income countr* OR middle-income countr*OR low income nation* OR middle income nation* OR lowincome nation* OR middle-income nation* OR low income world OR middle income world OR low-income world OR middle-income world OR low income econom*OR middle income econom* OR low-income econom* OR middle-income econom* 


\section{Search Searches \\ \#}

5.

Afghanistan[TIAB] OR Albania[TIAB] OR Algeria[TIAB] OR American Samoa[TIAB] OR

Angola[TIAB] OR Antigua[TIAB] OR Barbuda[TIAB] OR Argentina[TIAB] OR Armenia[TIAB] OR Azerbaijan[TIAB] OR Bangladesh[TIAB] OR Belarus[TIAB] OR Byelarus[TIAB] OR

Byelorussia[TIAB] OR Belorussia[TIAB] OR Belize[TIAB] OR Benin[TIAB] OR Bhutan[TIAB] OR Bolivia[TIAB] OR Bosnia[TIAB] OR Herzegovina[TIAB] OR Hercegovina[TIAB] OR BosniaHerzegovina[TIAB] OR Bosnia-Hercegovina[TIAB] OR Botswana[TIAB] OR Brazil[TIAB] OR Brasil[TIAB] OR Bulgaria[TIAB] OR Burkina[TIAB] OR Upper Volta[TIAB] OR Burundi[TIAB] OR Urundi[TIAB] OR Cambodia[TIAB] OR Republic of Kampuchea[TIAB] OR Cameroon[TIAB] OR Cameroons[TIAB] OR Cape Verde[TIAB] OR Central African Republic[TIAB] OR Chad[TIAB] OR Chile[TIAB] OR China[TIAB] OR Colombia[TIAB] OR Comoros[TIAB] OR Comoro Islands[TIAB] OR Comores[TIAB] OR Congo[TIAB] OR DRC[TIAB] OR Zaire[TIAB] OR Costa Rica[TIAB] OR Cote d'Ivoire[TIAB] OR Ivory Coast[TIAB] OR Cuba[TIAB] OR Djibouti[TIAB] OR Obock[TIAB] OR French Somaliland[TIAB] OR Dominica[TIAB] OR Dominican Republic[TIAB] OR Ecuador[TIAB] OR Egypt[TIAB] OR United Arab Republic[TIAB] OR El Salvador[TIAB] OR Eritrea[TIAB] OR Ethiopia[TIAB] OR Fiji[TIAB] OR Gabon[TIAB] OR Gabonese Republic[TIAB] OR Gambia[TIAB] OR Georgia[TIAB] OR Ghana[TIAB] OR Gold Coast[TIAB] OR Grenada[TIAB] OR Guatemala[TIAB] OR Guinea[TIAB] OR Guinea-Bissau[TIAB] OR Guiana[TIAB] OR Guyana[TIAB] OR Haiti[TIAB] OR Honduras[TIAB] OR India[TIAB] OR Indonesia[TIAB] OR Iran[TIAB] OR Iraq[TIAB] OR Jamaica[TIAB] OR Jordan[TIAB] OR Kazakhstan[TIAB] OR Kenya[TIAB] OR Kiribati[TIAB] OR Republic of Korea[TIAB] OR North Korea[TIAB] OR DPRK[TIAB] OR Kosovo[TIAB] OR Kyrgyzstan[TIAB] OR Kirghizstan[TIAB] OR Kirgizstan[TIAB] OR Kirghizia[TIAB] OR Kirgizia[TIAB] OR Kyrgyz[TIAB] OR Kirghiz[TIAB] OR Kyrgyz Republic[TIAB] OR Lao[TIAB] OR Laos[TIAB] OR Latvia[TIAB] OR Lebanon[TIAB] OR Lesotho[TIAB] OR Basutoland[TIAB] OR Liberia[TIAB] OR Libya[TIAB] OR Lithuania[TIAB] OR Macedonia[TIAB] OR Madagascar[TIAB] OR Malagasy Republic[TIAB] OR Malawi[TIAB] OR Nyasaland[TIAB] OR Malaysia[TIAB] OR Malaya[TIAB] OR Malay[TIAB] OR

Maldives[TIAB] OR Mali[TIAB] OR Marshall Islands[TIAB] OR Mauritania[TIAB] OR Mauritius[TIAB] OR Mayotte[TIAB] OR Mexico[TIAB] OR Micronesia[TIAB] OR Moldova[TIAB] OR Moldovia[TIAB] OR Mongolia[TIAB] OR Montenegro[TIAB] OR Morocco[TIAB] OR Mozambique[TIAB] OR Myanmar[TIAB] OR Burma[TIAB] OR Namibia[TIAB] OR Nepal[TIAB] OR Nicaragua[TIAB] OR Niger[TIAB] OR Nigeria[TIAB] OR Pakistan[TIAB] OR Palau[TIAB] OR Palestine[TIAB] OR Panama[TIAB] OR Papua New Guinea[TIAB] OR Paraguay[TIAB] OR Peru[TIAB] OR Philippines[TIAB] OR Romania[TIAB] OR Rumania[TIAB] OR Roumania[TIAB] OR Russia[TIAB] OR Russian Federation[TIAB] OR USSR[TIAB] OR Soviet Union[TIAB] OR Union of Soviet Socialist Republics[TIAB] OR Rwanda[TIAB] OR Ruanda-Urundi[TIAB] OR Samoa[TIAB] OR Samoan Islands[TIAB] OR Sao Tome[TIAB] OR Principe[TIAB] OR Senegal[TIAB] OR Serbia[TIAB] OR Montenegro[TIAB] OR Yugoslavia[TIAB] OR Seychelles[TIAB] OR Sierra Leone[TIAB] OR Solomon Islands[TIAB] OR Somalia[TIAB] OR South Africa[TIAB] OR Sri Lanka[TIAB] OR Ceylon[TIAB] OR Saint Kitts[TIAB] OR St Kitts[TIAB] OR Saint Christopher Island[TIAB] OR Nevis[TIAB] OR Saint Lucia[TIAB] OR St Lucia[TIAB] OR Saint Vincent[TIAB] OR St Vincent[TIAB] OR Grenadines[TIAB] OR Sudan[TIAB] OR Suriname[TIAB] OR Surinam[TIAB] OR Swaziland[TIAB] OR Syria[TIAB] OR Syrian Arab Republic[TIAB] OR Tajikistan[TIAB] OR Tadzhikistan[TIAB] OR Tadjikistan[TIAB] OR Tanzania[TIAB] OR Thailand[TIAB] OR Timor-Leste[TIAB] OR East Timor[TIAB] OR Togo[TIAB] OR Togolese Republic[TIAB] OR Tonga[TIAB] OR Tunisia[TIAB] OR Turkey[TIAB] OR Turkmenistan[TIAB] OR Turkmenia[TIAB] OR Tuvalu[TIAB] OR Uganda[TIAB] OR Ukraine[TIAB] OR Uruguay[TIAB] OR Uzbekistan[TIAB] OR Vanuatu[TIAB] OR New Hebrides[TIAB] OR Venezuela[TIAB] OR Vietnam[TIAB] OR Viet Nam[TIAB] OR West Bank[TIAB] OR Gaza[TIAB] OR Yemen[TIAB] OR Zambia[TIAB] OR Zimbabwe[TIAB] OR Rhodesia[TIAB]

6. 1 AND 2 AND 3 AND 4 AND 5

7. Limit 6 to humans 


\section{Screening and selecting studies}

Two authors will screen titles and abstract independently to find studies that meet the pre-specified eligibility criteria for inclusion in the review. Full texts of all potentially relevant studies will be accessed and assessed in detail in a similar manner. A third reviewer will be available to resolve any discrepancies between the two independent assessors. A screening guide will be used to ensure that independent reviewers apply the selection criteria reliably. Authors whose full-text documents are not available via a variety of internet-based sources will be contacted directly through the corresponding authors to provide them to help make the final decision about inclusion. If vital information needed to make the inclusion decision is not obtained, the article will be excluded. Mendeley reference manager will be used to deduplicate studies. Flowchart for full-text screening of search results adapted from AMIPS is shown in Fig. 1. The PRISMA flow chart for study selection is shown in Fig. 2.

\section{Data extraction and management}

Data such as first author's name, year of publication, study design, country, serological method (i.e. Lateral flow immunoassay (LFIA), Chemiluminescence immunoassay (CLIA), Enzyme-linked immunosorbent assay (ELISA), Virus neutralization assay and Microsphere immunoassay) total sample screened, seropositive people, and demographics (i.e. age, sex, etc.), economic status (Low income and Middle income), type of population (children, adolescents, adults) will be extracted from the included studies and recorded in a pre-tested and standardized excel spreadsheet. A second author will independently cross-check the extracted data for accuracy and completeness. All retrieved articles from the search will be managed and stored in the Mendeley reference manager.

\section{Dealing with missing data}

Missing data will not be imputed. Instead, lead authors of the primary studies will be contacted for missing, insufficient or unclear data. Where possible, we will ask the primary study authors to provide us with the raw data to extract the missing information.

\section{Quality assessment}

The Newcastle-Ottawa Quality Assessment tool adapted for observational and cross-sectional studies will be used to assess the quality of the included studies. The Newcastle-Ottawa Quality Assessment tool contains three domains that are graded on 10-point stars. Domain 1 evaluates the methodological quality of each study [5 stars], domain 2 assesses the comparability of the study [2 stars] and domain 3 evaluates the outcome measure and related statistical analysis [3 stars] [19]. Two independent reviewers will do the quality assessment, and the average will be taken as the final score for that particular study.

\section{Data synthesis and statistical analysis}

\section{Meta-analysis}

A simple descriptive overview and summary tables of the results of the included studies will be presented, organized around the SARS CoV-2 seroprevalence. Where considerable heterogeneity exists, DerSimonian and Laird random-effects model [20] will be used to pooled the seroprevalence of SARS CoV-2. First, we 
will estimate COVID-19 seroprevalence in individual continent such as (i.e., Africa, South America, Asia etc.). To estimate the number of people exposed to SARS-CoV-2, we will extrapolate seroprevalence estimates to the total human population living in that sub-region. If no heterogeneity is suspected, the fixed-effect model will be used for the effect estimates and visually represented using the forest plot.

For the associated risk factors for continuous SARS COV-2 in data LMICs that have been measured with the same scales, we will estimate the mean difference (MD) and their standard deviation (SD), reported with their $95 \% \mathrm{Cls}$. On the other hand, when different scales are used to measure the continuous outcome, we will use the standardized mean difference (SMD) and reported it with their $95 \%$ Cls. For dichotomous outcomes, we will estimate the pooled odds ratio (OR) or risk ratio (RR), depending on the nature of the outcomes, to determine the strength of association for the various indicators of seroprevalence using generic inverse-variance, reported with their $95 \% \mathrm{Cl}$.

\section{Heterogeneity and meta-regression}

Heterogeneity will be assessed by the $\mathrm{I}^{2}$ statistics, which measures the variation across studies due to heterogeneity rather than chance. $\left.\right|^{2}>50 \%$ will be classified as substantial heterogeneity. Cochrane's $Q$ statistics and Tau will also be presented and $p<0.05$ will be used as a cut-off for significant heterogeneity [21] A meta-regression will be conducted to identify the sources of heterogeneity in the pooled SARS CoV-2 seroprevalence estimate. Socio-demographics factors such as Sex, Age $(<12,12-17$ and $>18$ years) Type of population (high-risk population, i.e., health workers vs Low-risk population, i.e., general population, blood donors), serological method, continents, economic status (Low-income country and Middle-income country), continent (Africa, South America, Asia). A multivariable meta-regression model will be built by adding each variable sequentially starting with variables that shows strongest association with SARS CoV-2 seroprevalence in the univariate analysis. A P $\leq 0.2$ will be used to retain variables in the multivariable model.

\section{Sub-group analyses}

Where significant heterogeneity exists in the included studies, a subgroup analysis will be performed to determine the sources of heterogeneity based on the following: sex, age, type of population (high or lowrisk population), serological method, continents, economic status of the country and continent (Africa, South America, Asia etc.).

\section{Sensitivity analysis}

If possible, a sensitivity analysis will be performed to determine the robustness of the estimates obtained from the meta-analysis. We will do sensitivity analysis on the quality of the studies included in the systematic review and meta-analysis i.e., studies with low quality score will initially be excluded to check their direction and impact on the overall (pooled) estimate. Additionally, we will also use leave-one-out to check if any particular study/studies influence the pooled estimates (Ref:

https://online.stat.psu.edu/stat509/node/145/). 
For comparative studies, the analyses will be performed using Review Manager v 5.4 (The Cochrane Collaboration, Oxford, UK): https://training.cochrane.org/online-learning/core-software-cochrane-reviews, otherwise STATA 16 will be used for effect estimates and presented with the confidence interval.

\section{Discussion}

In 2020, the Coronavirus caused by Severe Acute Respiratory Syndrome Coronary virus-2 (SARS Cov-2) is the most significant public problem [1]. IgM is the first type of antibody developed by the body in response to an infection with COVID-19, followed by IgG, which replaces IgM as the predominant blood antibody [22]. Given the existence of asymptomatic cases and little access to diagnostic testing i.e. in LMICs, serological surveys are a valuable method to determine the magnitude of the epidemic [11].

To the best of our knowledge, this is the first study to explore and systematically review empirical evidence on SARS Cov-2 seroprevalence in LMICs. The study aims to comprehensively review and estimate the seroprevalence of SARS Cov-2 in individuals living in Low-and-Middle Income Countries (LMICs and to assess the risk factors for SARS Cov-2 infection in Low Middle Income Countries in the context of rolling out vaccines in these countries. The study could determine the extent of spread and exposure of SARS Cov-2 infection in LMICs. Due to regional and geographical differences, there can be variations across studies

\section{Strengths and limitations}

- To the best of the authors' knowledge, this is the first systematic review and meta-analysis on SARS Cov-2 Seroprevalence in LMICs. Also, during the registration process in PROSPERO, we did not come across any published or ongoing systematic review on this subject. PROSPERO Registration Number: CRD422020221548.

- The study could determine the extent of spread and exposure of SARS Cov-2 infection among persons living in LMICs and likely number to benefit from the imminent introduction of vaccines in LMICs.

- The search will be comprehensive and done in close collaboration with experienced medical librarians and will follow all good practices protocols for conducting and reporting systematic reviews.

- Due to regional and geographical differences, there can be variations across studies, therefore, we plan to conduct robust sub-group analyses to detect any sub-group effects. We will run metaregression analyses to identify any potentially useful trends and test the robustness of our effect estimates from sensitivity analyses.

- We have assembled world-class experts from diverse backgrounds and geographical locations, including epidemiology, evidence synthesis, economics, allied sciences, statistics, biomedicine, etc. to produce an innovative, demand-driven, policy and context-relevant systematic review is that will help guide policy and practice in the global control of COVID-19. 


\section{List Of Abbreviations}

CLIA - Chemiluminescence immunoassay

ELISA -Enzyme-linked immunosorbent assay

HCoVs - Human Corona Viruses

LFIA - Lateral flow immunoassay

LMICs - Low- and Middle-Income Countries

MD - mean difference

MOOSE - Meta-analysis of Observational studies in Epidemiology

OR - odds ratio

PRISMA-P - Preferred Reporting Items for Systematic review and Meta-analyses Protocol

PROSPERO - International Prospective Register of Systematic Reviews

RR - risk ratio

SARS - Severe Acute Respiratory Syndrome

SARS-CoV-2 - Severe Acute Respiratory Syndrome Coronavirus 2

SD - standard deviation

SMD - standardized mean difference

\section{Declarations}

\section{Ethics approval and consent to participate}

Ethical approval is not required as this is a secondary research and will use reported data in scientific literature. The results of this study will be made widely available and accessible to practitioners, patients and policy makers, and presented at relevant scientific conferences. A full manuscript will be submitted to a reputable peer-review journal for publication.

\section{Consent for publication}

Not applicable.

\section{Availability of data and materials}


All relevant data are within the manuscript.

\section{Competing interests}

I have read the journal's policy and the authors of this manuscript have the following competing interests: [Denny John was Adjunct Scientist, National Institute of Medical Statistics, Indian Council of Medical Research (ICMR), New Delhi, India, from Feb - Aug 2020. ICMR conducted a national sero survey of SARSCoV-2 from May 11-June 4, 2020. https://www.ijmr.org.in/article.asp?issn=09715916; ;ear $=2020$; volume $=152$;issue $=1$; spage $=48$;epage $=60$; aulast $=$ Murhekar. $]$. All other authors declare that there is no conflict of interest.

\section{Funding}

The author(s) received no specific funding for this work.

\section{Authors' Contributions}

MA - Conceptualization, data curation, Methodology, formal analysis, writing original draft, software LA - Data curation, Review and editing, Validation, Methodology

KOA - Review and Editing, Supervision, Validation, Resources, Project administration

NEW - Review and editing, validation

COY - writing original draft, Review and editing

RC - Data curation, Review and editing, Validation

DJ - Review and editing, Formal analysis

PE - Review and editing, validation

NT - Review and editing, validation

MGS - Review and editing, validation

ADA - Methodology, Data curation, review and editing, validation, Supervision

All authors read and approved the final manuscript.

\section{References}

1. Rostami A, Sepidarkish M, Leeflang M, Riahi SM, Shiadeh MN, Esfandyari S, et al. SARS-CoV-2 seroprevalence worldwide: a systematic review and meta-analysis. Clin Microbiol Infect [Internet]. 2020 (October 24); Available from: https://doi.org/10.1016/j.cmi.2020.10.020 
2. Paules Cl. Coronavirus Infections - More Than Just the Common Cold. JAMA. 2020;323(8):707-8.

3. Shah VK, Firmal P, Alam A, Ganguly D, Chattopadhyay S. Overview of Immune Response During SARS-CoV-2 Infection: Lessons From the Past. Front Immunol. 2020;11:1-17.

4. Lim Y, Ng Y, Tam J, Liu D. Human Coronaviruses: A Review of Virus-Host Interactions. Diseases. 2016;4(4):26.

5. Wang C, Pan R, Wan X, Tan Y, Xu L, Ho CS, et al. Immediate Psychological Responses and Associated Factors during the Initial Stage of the 2019 Coronavirus Disease ( COVID-19) Epidemic among the General Population in China. Int J Environ Res Public Health. 2020;17:1-25.

6. Rieg S, von Cube M, Kalbhenn J, Utzolino S, Pernice K, Bechet L, et al. COVID-19 in-hospital mortality and mode of death in a dynamic and non-restricted tertiary care model in Germany. PLoS One [Internet]. 2020;15(11 November):1-16. Available from:

http://dx.doi.org/10.1371/journal.pone.0242127

7. Verity R, Okell LC, Dorigatti I, Winskill P, Whittaker C, Imai N, et al. Estimates of the severity of coronavirus disease 2019: a model-based analysis. Lancet Infect Dis. 2020;20(6):669-77.

8. Oran DP, Topol EJ. Prevalence of Asymptomatic SARS-CoV-2 Infection: A Narrative Review. Ann Intern Med. 2020;173(5):362-7.

9. Tsitsilonis OE, Paraskevis D, Lianidou E, Pierros V, Akalestos A, Kastritis E, et al. Seroprevalence of antibodies against SARS-CoV-2 among the personnel and students of the national and kapodistrian university of athens, greece: A preliminary report. Life. 2020;10(9):1-8.

10. Anand S, Montez-Rath M, Han J, Bozeman J, Kerschmann R, Beyer P, et al. Prevalence of SARS-CoV-2 antibodies in a large nationwide sample of patients on dialysis in the USA: a cross-sectional study. Lancet [Internet]. 2020;396(10259):1335-44. Available from: http://dx.doi.org/10.1016/S01406736(20)32009-2

11. Pollán M, Pérez-Gómez B, Pastor-Barriuso R, Oteo J, Hernán MA, Pérez-Olmeda M, et al. Prevalence of SARS-CoV-2 in Spain (ENE-COVID): a nationwide, population-based seroepidemiological study. Lancet. 2020;396(10250):535-44.

12. Poland GA, Ovsyannikova IG, Kennedy RB. SARS-CoV-2 immunity: review and applications to phase 3 vaccine candidates. Lancet [Internet]. 2020;396(10262):1595-606. Available from: http://dx.doi.org/10.1016/S0140-6736(20)32137-1

13. Huang AT, Garcia-Carreras B, Hitchings MDT, Yang B, Katzelnick LC, Rattigan SM, et al. A systematic review of antibody mediated immunity to coronaviruses: kinetics, correlates of protection, and association with severity. Nat Commun [Internet]. 2020;11(1):1-16. Available from: http://dx.doi.org/10.1038/s41467-020-18450-4

14. Spellberg B, Nielsen TB, Casadevall A. Antibodies, Immunity, and COVID-19. JAMA Intern Med. 2020;19-20.

15. Stout RL, Rigatti SJ. The Seroprevalence of SARS-CoV-2 Antibody in the Asymptomatic Population. medRxiv Prepr. 2020; Published online 6 December. doi.org/10.1101/2020.11.10.20215145 (Preprint) 
16. Chibwana, Marah G, Khuzwayo C. Jere, Raphael Kamn'gona JM. High SARS-CoV-2 seroprevalence in health care workers but relatively low numbers of deaths in urban Malawi. medRxiv. 2020; Published online 5 August. https://doi.org/10.1101/2020.07.30.20164970. (Preprint)

17. Shamseer L, Moher D, Clarke M, Ghersi D, Liberati A, Petticrew M, et al. Preferred reporting items for systematic review and meta-analysis protocols (prisma-p) 2015: Elaboration and explanation. BMJ [Internet]. 2015;349(January):1-25. Available from: http://dx.doi.org/doi:10.1136/bmj.g7647

18. Stroup DF, Berlin JA, Morton SC, Olkin I, Williamson G, Moher D, et al. Meta-analysis of Observational Studies in Epidemiology: A Proposal for Reporting - Meta-analysis Of Observational Studies in Epidemiology (MOOSE) Group B. JAMA Neurol. 2000;283:2008-12.

19. Luchini C, Stubbs B, Solmi M, Veronese N. Assessing the quality of studies in meta-analyses: Advantages and limitations of the Newcastle Ottawa Scale. World J Meta-Analysis. 2017;5(4):80.

20. DerSimonian R, Laird N. Meta-analysis in clinical trials revisited. Contemp Clin Trials [Internet]. 2015;45:139-45. Available from: http://dx.doi.org/10.1016/j.cct.2015.09.002

21. Higgins JPT, Thompson SG. Quantifying heterogeneity in a meta-analysis. Stat Med. 2002;21(11):1539-58.

22. Wajiha Javed, Jaffer Bin Baqar, Syed Hussain Baqar Abidi WF. Sero-prevalence Findings from Metropoles in Pakistan: Implications for Assessing. medRxiv Prepr. 2020; Published online 14 August. doi.org/10.1101/2020.08.13.20173914 (Preprint)

\section{Supplementary Files}

This is a list of supplementary files associated with this preprint. Click to download.

- Supplementaryfile129122020.docx

- Supplementaryfile229122020.docx 